\title{
Evaluación de la eficacia de la guía quirúrgica ROGA y la técnica a mano alzada en el posicionamiento tridimensional del implante en pacientes del Centro Odontológico de la Universidad Católica de Santa María de Arequipa 2019.
}

Evaluation of the efficacy of the ROGA surgical guide and the freehand technique in the three-dimensional positioning of the implant in patients of the Centro Odontológico de la Universidad Católica de Santa María de Arequipa 2019.

\section{Roxana Gamarra Ojeda y Alberto Cáceres Huambo}

Universidad Nacional de Santa María. Arequipa Perú.

\section{INFORMACIÓN}

\section{Historia del Artículo}

Recepción: 06/12/2019

Revisión: 18/02/2020

Aceptación: 20/05/2020

\section{Palabras Clave \\ guía quirúrgica, implantes,} posicionamiento tridimensional.

\section{Key Words}

surgical guide, implants, threedimensional positioning

\section{DOI}

https://doi.org/10.35286/veritas. v21i 2.278

\begin{abstract}
RESUMEN
Eléxito de una rehabilitación sobre implantes radica en el correcto posicionamiento tridimensional del implante y una guía quirúrgica que ha sido previamente planeada con un protocolo reveso nos acerca a ese objetivo; se crea la guía quirúrgica ROGA que pretende ser más eficaz que la técnica a mano alzada en dicho posicionamiento; para evaluarla se colocó 8 implantes con Guía ROGA y 8 implantes a mano alzada, y luego se evaluó el posicionamiento tridimensional utilizando las tomografías del antes y después, se evaluaron 5 medidas, mesiodistal y vestíbulo palatina ambas a nivel coronal y a nivel apical y la ápicocoronal; los implantes colocados con la guía ROGA no tuvieron ninguna desviación a nivel mesiodistal coronal y en las demás medidas tuvieron menos desviaciones con relación a la planificación reversa con una media de $0,457 \mathrm{~mm}$ a diferencia de la de mano alzada que tuvo una desviación media de hasta $1,91 \mathrm{~mm}$; se encontró diferencia estadísticamente significativo en las demás medidas no hubo diferencia estadísticamente significativo. Sin embargo, en implantes una pequeña desviación milimétrica puede afectar el tratamiento rehabilitador. Se concluyó que la guía ROGA es más eficaz en el posicionamiento tridimensional del implante dental.
\end{abstract}

\section{INTRODUCCIÓN}

La implantología es un pilar importante en la odontología general con la que devolvemos la salud bucal, la función y estética perdidas por falta o extracción de piezas dentarias.

Mayormente para la colocación de los implantes se usa la técnica de mano alzada, esta técnica en manos de un cirujano experto puede tener buenos resultados, pero en personas que recién ingresan al mundo de la implantología puede traer complicaciones, unas a nivel de la rehabilitación y otras

Correspondencia:

Roxana Gamarra Ojeda rgamarra@ucsm.edu.pe complicaciones más serias porque se perforó alguna tabla ósea o se ingresó a un reparo anatómico. Existe muchos tipos de guía, la guía computarizada CAD CAM permite guiar el recorrido de toda la fresa, a diferencia de otras guías que solo nos permiten conocer el sitio donde se hará la primera perforación, pero esta es aun costosa para nuestro medio, además que para su uso se requiere otros gastos como un juego de fresas especial, cilindros de titanio y costos guía digital y la impresa en $3 \mathrm{D}$; se han hecho estudios que indican que este tipo de guía recalienta el lecho óseo ya que no permiten la refrigeración de las brocas; por ello se confeccionó la guía quirúrgica ROGA, que es económica, reutilizable, autoclavable, no impide la refrigeración de la fresa ya que el plano guía está a nivel del cabezal del contrángulo y no requiere fresas especiales. 


\section{MATERIAL Y MÉTODOS}

Estudio cuasiexperimental, observacional y transversal. Se seleccionaron 16 pacientes que cumplían los criterios de inclusión y se los dividió al azar en dos grupos, un grupo al que se le colocaría sus implantes de la manera tradicional a mano alzada y el otro grupo que recibiría la colocación de implantes usando la guía ROGA, en ambos casos se les realizó la $\mathrm{HC}$, firma del consentimiento informado, modelos de estudio, encerado de diagnóstico, basado en este se confeccionó la guía tomográfica, y se tomó la tomografía inicial, se hizo las mediciones del reborde óseo residual escogiéndose el diámetro y longitud del implante y se hizo la simulación digital del implante. Con los pacientes que recibirían con la guía ROGA se pasa al trabajo laboratorio que consiste en hacer un mapping en el modelo troquelado del sitio del implante y luego se posiciona la guía con la plataforma que guía la fresa en la posición que se determine luego del análisis del protocolo reverso. Luego con la guía posicionada adecuadamente se va a la parte quirúrgica y se coloca el implante con ayuda de la misma. A los 7 días de colocado el implante se toma la tomografía final para evaluar la posición final. Luego se evalúa el desplazamiento que tuvo el implante en las 5 medidas mesiodistal coronal y apical, vestíbulo palatino o lingual corona y apical y la medida ápicocoronal; todo esto con el planeamiento digital en la primera radiografía y la tomografía final en ambos casos con guía ROGA y con la técnica a mano alzada. Los datos recolectados fueron registrados en una ficha, estos fueron codificados para un mejor procesamiento, se utilizó el software IBM SPSS Statistic 23 para la contrastación de la hipótesis con una prueba de t_de students y Kruskal Wallis así mismo se calcularon las medias y las desviaciones estándar.

\section{RESULTADOS}

EFICACIA DE LA GUIA QUIRÚRGICA ROGA EN EL POSICIONAMIENTOTRIDIMENSIONAL DEL IMPLANTE UNITARIO

Tabla 1: Medidas descriptivas de los casos con Guía Quirúrgica ROGA. Fuente: Matriz de datos

\begin{tabular}{ccccc}
\hline \multicolumn{5}{c}{ Desviación } \\
\hline MEDIDAS & Media & $\begin{array}{c}\text { Desviación } \\
\text { estándar }\end{array}$ & Mínimo & Máximo \\
\hline MD_coronal & 0 & 0 & 0 & 0 \\
MD_apical & 0.3286 & 0.56188 & 0 & 1.2 \\
VPL_coronal & 0.4571 & 0.61875 & 0 & 1.4 \\
VPL_apical & 0.4143 & 0.47056 & 0 & 1.2 \\
Corona_apical & 1.5429 & 1.56403 & 0 & 4 \\
\hline
\end{tabular}

Tabla 2: Porcentajes de la dirección de la desviación de los casos en Guía Quirúrgica ROGA. Fuente: Matriz de datos

\begin{tabular}{|c|c|c|c|c|c|}
\hline Dirección & $\mathrm{s} / \mathrm{d}$ & mesial & distal & vestibular & $\begin{array}{c}\text { palatino o } \\
\text { lingual }\end{array}$ \\
\hline MD_coronal & $100 \%$ & & & & \\
\hline MD_Apical & $71.40 \%$ & & $28.60 \%$ & & \\
\hline VP_Coronal & $57.10 \%$ & & & $42.90 \%$ & \\
\hline VP_Apical & $42.90 \%$ & & & $28.60 \%$ & $28.60 \%$ \\
\hline
\end{tabular}

Tabla 3: Medidas descriptivas de los casos a mano alzada. Fuente: Matriz de datos

\begin{tabular}{ccccc}
\hline \multicolumn{5}{c}{ Desviación } \\
\hline MEDIDAS & Media & estándar & Mínimo & Máximo \\
\hline MD_coronal & 0.575 & 1.12853 & 0 & 3 \\
MD_apical & 1.9125 & 1.56336 & 0 & 5.2 \\
VPL_coronal & 0.8125 & 0.58904 & 0 & 17 \\
VPL_apical & 1.2 & 0.91496 & 0 & 2.7 \\
Corono_apical & 0.475 & 0.9573 & -1 & 1.8 \\
\hline
\end{tabular}

Tabla 4: Porcentajes de la dirección de la desviación en los casos a mano alzada. Fuente: Matriz de datos

\begin{tabular}{cccccc}
\hline Dirección & $\mathrm{s} / \mathrm{d}$ & mesial & distal & vestibular & $\begin{array}{c}\text { palatino o } \\
\text { lingual }\end{array}$ \\
\hline MD_coronal & $75 \%$ & & $25 \%$ & & \\
MD_Apical & $12.50 \%$ & $12.50 \%$ & $75.00 \&$ & & \\
VP_Coronal & $25.00 \%$ & & & $62.50 \%$ & $12.50 \%$ \\
VP_Apical & $12.50 \%$ & & & $37.50 \%$ & $50.00 \%$ \\
\hline
\end{tabular}

En la tabla 1 con la Guía ROGA vemos que en la medida mesiodistal a nivel coronal no existe desviación, en las demás medidas la media mayor es de $0,457 \mathrm{~mm}$, solo en la medida ápicocoronal se tiene una media de $1.54 \mathrm{~mm}$.

En la tabla 2 nos indica que el $28.60 \%$ se desvía hacia distal, los demás casos no se desvían; en la dirección vestíbulo palatino o lingual se desvían hacia vestibular en un $42.90 \%$ los demás casos o no se desvían o lo hacen a palatino o lingual.

\section{EFICACIA DE LA TÉCNICA A MANO ALZADA EN ELPOSICIONAMIENTOTRIDIMENSIONAL DEL IMPLANTE UNITARIO}

En la tabla 3 las mayores desviaciones las encontramos en las medidas apicales con una media de $1,912 \mathrm{~mm}$ en la mesiodistal y de 1.2 en el vestíbulo palatina o lingual.

En la tabla 4 vemos que el $75 \%$ de los casos se en la medida mesiodistal se fueron hacia distal, y en la vista vestíbulo palatina o lingual el $62 \%$ a nivel coronal se fueron hacia vestibular, pero en apical se fueron el $50 \%$ hacia palatino o lingual. 


\section{DIFERENCIA O SIMILITUD EN LA EFICACIA DE AMBOS SISTEMAS EN LA COLOCACION DEL IMPLANTE UNITARIO.}

Tabla 5: Comparación del uso de Guía ROGA y la Técnica a mano alzada en la media Mesiodistal. Fuente: Matriz de datos

\begin{tabular}{ccccc}
\hline \multirow{2}{*}{$\begin{array}{c}\text { Estadísticos } \\
\text { descriptivos }\end{array}$} & \multicolumn{2}{c}{ MD-CORONAL } & \multicolumn{2}{c}{ MD-APICAL } \\
\cline { 2 - 5 } & SIN GUIA & CON GUIA & SIN GUIA & CON GUIA \\
\hline Mínimo & 0 & 0 & 0 & 0 \\
Máximo & 3 & 0 & 5.2 & 1.2 \\
Media & 0.575 & 0 & 1.9125 & 0.3286 \\
$\begin{array}{c}\text { Desviación } \\
\text { Estándar }\end{array}$ & 1.1285 & 0 & 1.563 & 0.5619 \\
\hline
\end{tabular}

Tabla 6: Comparación del uso de Guía ROGA y la Técnica a Mano Alzada en la medida Vestíbulo Platino/ lingual. Fuente: Matriz de datos.

\begin{tabular}{ccccc}
\hline & \multicolumn{2}{c}{ VP CORONAL } & \multicolumn{2}{c}{ VP APICAL } \\
\cline { 2 - 5 } & SIN GUIA & CON GUIA & SIN GUIA & CON GUIA \\
\hline Media & 0.8125 & 0.4571 & 1.2 & 0.4143 \\
Mínimo & 0.0000 & 0.0000 & 0.000 & 0.0000 \\
Máximo & 1.70000 & 1.4000 & 2.700 & 1.2000 \\
$\begin{array}{c}\text { Desviación } \\
\text { Estándar }\end{array}$ & 0.58904 & 0.61875 & 0.91496 & 0.47056 \\
\hline
\end{tabular}

Los gráficos 1 y 2 muestra la diferencia en las dos técnicas, línea azul es el nivel de desviación que tuvieron los implantes con guía ROGA se puede ver que tuvo menor desviación que la técnica a mano alzada línea de color naranja.

El valor $\mathrm{p}$ a nivel coronal sale 0.171 esta es mayor a 0.05 no hay diferencia estadísticamente significativa, en la medida mesio_distal a nivel apical el valor $p$ fue de 0.010 menor a 0.05 si existe diferencia estadísticamente.

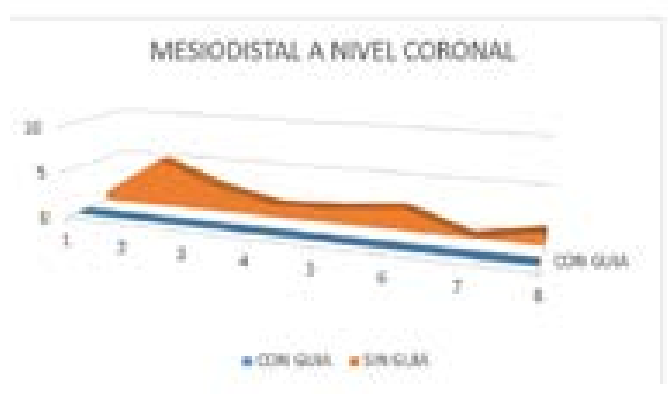

Gráfico 1: Medida mesiodistal a nivel coronal con guía ROGA y a mano alzada

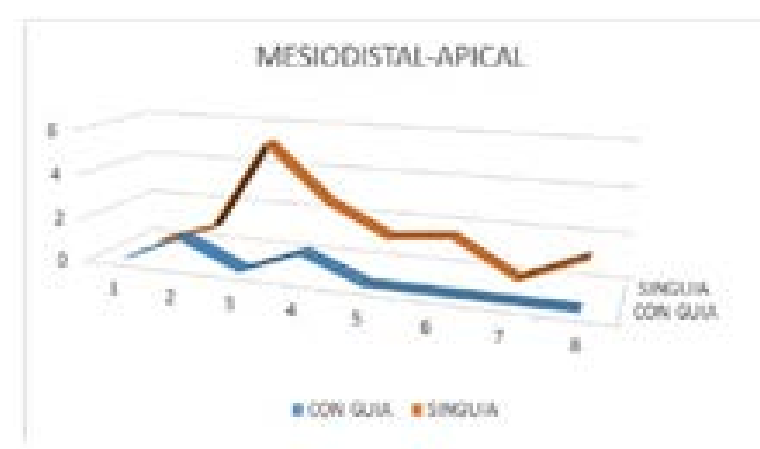

Gráfico 2: Medida mesiodistal a nivel apical con guía ROGA y a mano alzada
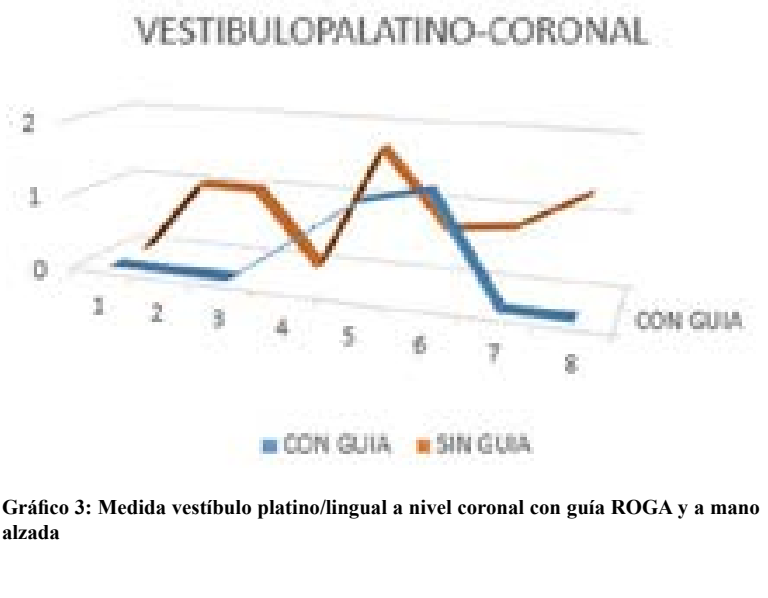

VESTIBULOPALATINO-APICAL

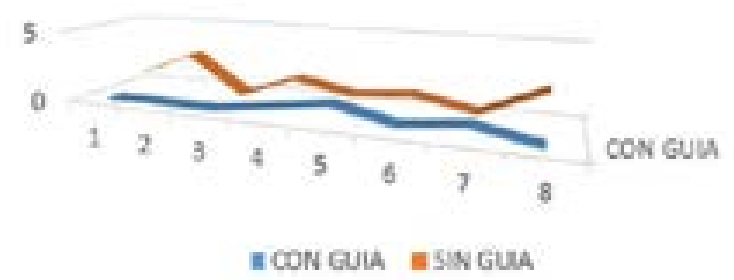

Gráfico 4: Medida vestíbulo platino/lingual a nivel apical con guía ROGA y a mano alzada

Las medidas VP a nivel coronal no pasa la prueba de normalidad y se realiza la prueba de kruskal wallis $\mathrm{p}<0.05 \%$ La Medida vestíbulo/palatina-lingual a nivel coronal con la prueba Kruskal wallis nos sale 0,188 esta es mayor a 0.05 por lo que se conserva la hipótesis nula es decir que no hay diferencia estadísticamente significativa en esta medida.

$\mathrm{P}<0.05 \%$ La Medida vestíbulo/palatina-lingual a nivel apical si pasa la prueba de normalidad por lo que se usa la prueba $t$ de students. Con la prueba $t$ de student nos da 0.062 esta es mayor a 0.05 por lo que se conserva la hipótesis nula es decir que no hay diferencia estadísticamente significativa en esta medida.

En ambas medidas vestíbulo palatina o lingual tanto coronal como apical con las pruebas estadísticas las diferencias son estadísticamente similares, pero como se dijo anteriormente en implantología los milímetros son importantes por ello al ver los datos si se ven diferencias y 
demostrándonos a través de números que la técnica con la guía ROGA es superior a la técnica a mano alzada.

En las medidas vestíbulo palatino a nivel coronal y apical los $\mathrm{p}$ valor fueron mayores a 0.05 por lo que no son estadísticamente significativos.

Tabla 7: Determinar el posicionamiento del implante en el sentido apicoronal con y sin Guía quirúrgica ROGA en relación al planeamiento tomográfico del implante

\begin{tabular}{cccccc}
\hline & Tipo_guía & N & Media & $\begin{array}{c}\text { Desviación } \\
\text { estándar }\end{array}$ & $\begin{array}{c}\text { Media } \\
\text { de error } \\
\text { estándar }\end{array}$ \\
\hline \multirow{2}{*}{ Corono_apical } & S/G & 8 &, 4750 &, 95730 &, 33846 \\
& $\mathrm{C} / \mathrm{G}$ & 7 & 1,5429 & 1,56403 &, 59115 \\
\hline
\end{tabular}

En este cuadro vemos que la media de desviación apicoronal en el grupo sin guía de $0.475 \mathrm{~mm}$ y en el con guía es de 1.54 esto nos indica que en el grupo sin guía estuvieron mejor posicionados apicocoronalmente, sin embargo, este parámetro no depende de la guía si no de la habilidad del cirujano.

Al realizarse la prueba t de students nos dio de 0.129 esta es mayor a 0,05 por lo cual se concluye que no hay diferencia estadísticamente significativa entre ambos casos.

\section{DISCUSIÓN}

La fabricación de una guía quirúrgica adecuada es fundamental para el éxito de las restauraciones de implantes. La mayoría de guías quirúrgicas solo enfatizan el sitio de la perforación y no guían todo el camino de la fresa, salvo la guía computarizada CAD_CAM. (1).

Se sugirió que las guías hechas con CAD CAM mejorarían la precisión de la planificación y colocación de implantes dentales, sin embargo no hay evidencia definitiva y la técnica implica gastos adicionales por ello (2) Giovani J en su estudio Clinical Application of Stereolithographic Surgical Guides for Implant Placement: Preliminary Results, verificó el grado de desplazamiento de los implantes colocados con este tipo de guías y encontró desviaciones a nivel coronal que van de $1.45 \pm 1.42 \mathrm{~mm}$, y a nivel apical de $2.99 \pm 1.77 \mathrm{~mm}$.

En nuestro estudio las desviaciones fueron menores a 0,5 $\mathrm{mm}$. Pero la forma como midió las desviaciones fue igual a nuestro estudio Figura 01.
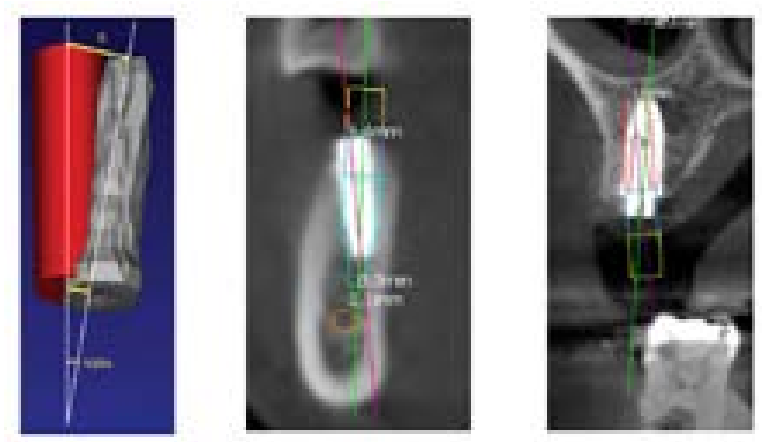

Fig.1: Método de verificar la desviación
Besimo y colaboradores (3) también evaluó el grado de desviación de los implantes con guías CAD CAM, y guías convencionales encontrando en las convencionales 0,9 a $1 \mathrm{~mm}$ en apical de la guía computarizada, y desviaciones de 1,5 y 2,1 en apical de las guías convencionales. Choi Mijin 2004, indicó que algunas guías solo permiten el taladro de la fresa helicoidal de $2 \mathrm{~mm}$ y solo es la inicial y el cirujano debe ir todas las demás fresas a mano alzada, y eso afecta la precisión de la dirección del implante, por ello la mayor desviación es a nivel apical como sucedió también con la guía ROGA y la técnica a mano alzada. (4)

Como vemos a pesar que la creación de prototipos de guías asistida por ordenador puede ser útil en la colocación de implantes, la técnica requiere una mejora para proporcionar una mejor estabilidad de la guía durante la cirugía, de igual forma en nuestra guía hace falta mejorar la estabilidad.

\section{CONCLUSIONES}

1. La Guía Quirúrgica ROGA ha demostrado ser eficaz en el $100 \%$ de los casos en la medida mesiodistal a nivel coronal, y en las demás medidas tiene un desplazamiento de menos de medio milímetro, y en la posición ápico coronal tuvo un desplazamiento mayor $1.5429 \mathrm{~mm}$.

2. Con la técnica a mano alzada se vió un desplazamiento de la medida mesio distal a nivel apical de casi dos milímetros $1.9125 \mathrm{~mm}$, en la medida mesiodistal a nivel coronal la desviación media fue de 0.575 , y la medida vesstibulo palatina coronal $0.8125 \mathrm{~mm}$ y apical $1.2 \mathrm{~mm}$ y apico coronal fue de $0.475 \mathrm{~mm}$ respectivamente.

3. Al comparar ambas técnicas se vió que las desviaciones con la guia quirúrgica ROGA fueron menores a la tecnica a mano alzada esto al evaluar sus medias y en la medida mesiodistal a nivel apical hubo un $p$ valor de 0,010 que nos indica que en esta medida la guia quirurgica ROGA y la técnica de mano alzada tuiveron una diferencia estadísticamente significativa. Se concluye que la guia quirúrgica ROGA es más eficaz que la técnica a mano alzada en el posicionamiento tridimensional del implante dental, en el centro odontológico de la UCSM, Arequipa 2019.

\section{AGRADECIMIENTOS}

Al Vicerectorado de Investigación y el personal que trabaja en el por su constante apoyo

\section{REFERENCIAS BIBLIOGRÁFICAS}

1. DixonDL BL. Surgical guide fabrication for an angled implant. J Prosthet Dent. 1996; p. 75:562-5.

2. Giovanni A.P. Di Giacomo. Clinical Application of stereolithographic surgical guides for implant placement: preliminary results. J Periodontol. 2005 April.

3. Besimo CE LJGJ. Accuracy of implant treatment planning utilizing template-guided. Dentomaxillofac Radiol. 2000 Abril; 29(46-51).

4. Choi Mijin RE,CD. Effects of varied dimensions of surgical guides on implant angulations. J prosther Dent. 2004; p. 463-9. 